\title{
Rapid Increase of a SAR-CoV-2 (COVID-19) Variant with Multiple Spike Protein Mutations
}

\author{
Cheepsattayakorn $\mathbf{A}^{1,2 *}$, Cheepsattayakorn $\mathbf{R}^{3}$ and Siriwanarangsun $\mathbf{P}^{1}$ \\ ${ }^{1}$ Faculty of Medicine, Western University, Pathumtani Province, Thailand \\ ${ }^{2} 10^{\text {th }}$ Zonal Tuberculosis and Chest Disease Center, Chiang Mai, Thailand \\ ${ }^{3}$ Department of Pathology, Faculty of Medicine, Chiang Mai University, Chiang Mai, Thailand
}

Editorial

Volume 6 Issue 1

Received Date: January 09, 2021

Published Date: January 19, 2021

*Corresponding author: Attapon Cheepsattayakorn, 10 $10^{\text {th }}$ Zonal Tuberculosis and Chest

Disease Center, 143 Sridornchai Road, Changklan Muang, Chiang Mai 50100, Thailand, Tel: 6653140767; 6653276364; Fax: 6653140773; 6653273590; Email: Attapon1958@gmail.com

\section{Editorial}

The new variant of SARS-CoV-2 (COVID-19), picked up by the COVID-19 Genomics UK (COG-UK) consortium that defined by multiple spike protein mutations (D1118H, S982A, T716I, P681H, D614G, A570D, N501Y, deletion 144, deletion 69-70) as well as mutations in other genomic regions was present in the South-East England during the late December 2020. With an estimated potential to increase the reproductive number (R) by at least 0.4 with an estimated increased transmissibility of up to $70 \%$, this COVID-19 variant is significantly more transmissible than previously circulating COVID-19 variants. Currently, there is no indication of increased infection severity observed associated with this COVID-19 variant, but the challenging assessment is that the majority of reported patients were under 60 years old, who are less likely to develop severe symptoms.
None of the previously identified SARS-CoV-2 variant have been demonstrated to cause increased infection severity. In Singapore, a clade 19B variant with lower infection severity was identified in the Spring and then disappeared. This new COVID-19 variant have been reported in Denmark, the Netherland, including a family of 4 Britons who arrived from Kent, South-East England, and are currently in quarantine at a private hospital in Bangkok, Thailand.

\section{Conclusion}

In conclusion, any increased transmissibility would increase the likelihood of spread, especially if increased family and social mixing is not decreased, and further spread outside the UK, particularly if non-essential travel is not decreased. 